\title{
Identifying the Impacts of Strategic Wind Farm Maintenance on its Capacity Value
}

\author{
David H. Kiefte ${ }^{1}$, Nathaniel S. Pearre ${ }^{1}$, Lukas G. Swan ${ }^{1}$ \\ ${ }^{1}$ Mechanical Engineering, Dalhousie University, Halifax, Canada \\ *David.Kiefte@dal.ca
}

\begin{abstract}
Wind farms in Nova Scotia create revenue based on the energy generated, independent of the time of delivery. Most wind farm operators schedule their maintenance during low wind resource periods to maximize production/revenue. These low resource periods can coincide with high electrical load periods which is disadvantageous to the utility supplying that load. A certain wind farm in Nova Scotia attempts to avoid scheduled maintenance during these high-load periods. We evaluate the output of this wind farm compared to other Nova Scotia wind farms to see if this strategy improves the "capacity value" by generating during high load periods. The wind farms span much of mainland Nova Scotia.

Data was collected from Supervisory Control and Data Acquisition (SCADA) systems on wind turbines and electrical substations. We also obtained total provincial electrical load from the electricity utility. The data were first normalized by dividing time-series production by the rated capacity of each farm. To examine the reliable capacity of each wind farm, normalized production during the highest $10 \%$ of load times was characterized by its $10^{\text {th }}$ percentile, $5^{\text {th }}$ percentile, and $1^{\text {st }}$ percentile production, i.e., the production available on at least $90 \%, 95 \%$, and $99 \%$ of such high-load times. This procedure was then repeated for the $5 \%$ highest load times, $1 \%$ and $0.5 \%$, to see how the data behaved as the range shortened.

Reliable capacity during high load periods is of interest to policy makers and electricity planners. We find that the reliable capacity of all of the wind farms increased dramatically as analysis focused on progressively higher and higher load times. This suggests that high winds contribute to provincial loads, which is likely related to wind increasing convection and cold air intrusion in buildings, so increasing heating loads. However, the analysis found that the specific wind farm with strategic scheduling of maintenance does not offer significantly better reliability than the other wind farms. The impact of strategic maintenance is not large enough relative to exogeneous differences between wind farms to result in better production during high load times. We conjecture that wind farm location has a higher impact than maintenance timing, and that a correlation between high wind speeds and high loads makes it an algorithmic distinction without an operational difference.
\end{abstract}

Word count: 370 